\title{
3D MULTI-RESOLUTION MAPPING OF MARS USING CASP-GO ON HRSC, CRISM, CTX AND HIRISE
}

\author{
Jan-Peter Muller ${ }^{1 *}$, Yu Tao ${ }^{1}$, Alfiah Ritzky Diana Putri ${ }^{1}$, Susan J. Conway ${ }^{2}$ \\ ${ }^{1}$ Imaging group, Mullard Space Science Laboratory, Dept. of Space and Climate Physics, University College London, \\ Holmbury St Mary, Dorking, Surrey, UK - (j.muller, yu.tao, alfiah.putri.15)@ucl.ac.uk \\ ${ }^{2}$ Laboratoire de Planétologie et Géodynamique, 2 rue de la Houssinière, Nantes, France - susan.conway@univ-nantes.fr
}

Planetary Remote Sensing and Mapping (III/II)

KEY WORDS: Digital Terrain Model, 3D, HiRISE, CTX, HRSC, CaSSIS, MOLA, CASP-GO, Valles Marineris.

\begin{abstract}
:
Automated large-scale retrieval of stereo photogrammetric DTMs of Mars fall into three categories: use of COTS software such as BAE-SOCET ${ }^{\circledR}$; private software such as the DLR-VICAR software suite and open source solutions such as the NASA Ames Stereo Pipeline (ASP). We describe here a novel open source system developed on the back of ASP known as CASP-GO (Tao et al., 2018) which has automated and extended ASP to be able to be applied to all modern single-pass or repeat-pass stereo photogrammetric datasets from $21^{\text {st }}$ century systems such as HRSC, CTX and HiRISE, CASP-GO also includes an automated co-registration system which employs HRSC (itself linked to MOLA) as the base-map upon which all other DTMs are co-registered. We show an example here of this automated co-registration system applied to multi-resolution stacks including CRISM images. Several thousand multiresolution 3D products, Digital Terrain Models (DTMs) and their corresponding orthorectified images (ORIs) have been generated and used in a wide variety of scientific studies, a few examples of which are shown here. Finally, a new method distributing these products providing long-term archiving and ease of access using DOIs is shown employing the ESA-PSA Guest Storage Facility and their corresponding display within the iMars webGIS system.
\end{abstract}

\section{INTRODUCTION}

3D modelling is essential to improving our understanding of the formation and erosional processes of the Martian surface. The rapid progress in planetary surface reconnaissance instrumentation, especially in relation to $3 \mathrm{D}$ imaging of the surface, has allowed different geologies, processes, features, and phenomena to be studied. Over the last 5 decades, Mars have been thoroughly imaged with serendipitous or planned stereo pairs at different spatial resolutions.

Previously, within the EU FP-7 iMars (http://www.i-mars.eu) project, a fully automated multi-resolution Digital Terrain Model (DTM) processing chain was developed at UCL for Mars Reconnaissance Orbiter (MRO) Context Camera (CTX), High Resolution Imaging Science Experiment (HiRISE) and Mars Express High-Resolution Stereo Camera (HRSC) stereo-pairs, called the Co-registration ASP with Gotcha Optimisations (CASP-GO) [1]. We show here a variety of different new products generated using CASP-GO, including large-area mosaics and introduce the reader to a new method of distribution of these products at the ESA-GSF.

\section{METHODS}

The CASP-GO pipeline is based on the NASA ASP framework [2] with specific enhancements, dealing with matching artefacts, gaps, and co-alignment issues, from in-house software previously developed in the Imaging Group at UCL-MSSL. The CASP-GO system [1] guarantees global areo-referencing congruence with respect to the aerographic coordinate system defined by HRSC, level-4 products (50-100m DTM grids) and thence to the Mars Orbiter Laser Altimeter (MOLA), providing much higher

\footnotetext{
$1 *$ corresponding author
}

resolution stereo derived DTMs than previously available, especially with CTX (18m grids) and HiRISE (1m DTM grids).

The overall CASP-GO workflow for DTM production includes the following 10 steps which are shown in Figure 1 and are described as follows: (a) ASP image pre-processing including image enhancement (e.g., Laplacian of Gaussian filtering and image normalisation) and stereo rectification; (b) ASP disparity map initialisation using coarse cross-correlation; (c) UCL fast Maximum Likelihood (f-ML) matching and the construction of an initial "float" disparity map; (d) ASP Bayes Expectation Maximisation (EM) weighted affine adaptive sub-pixel crosscorrelation; (e) UCL re-defined outlier rejection and gap erosion scheme to detect, remove and eliminate mis-matched and unreliable disparity values; (f) UCL ALSC (adaptative Least Squares Correlation called Gotcha) sub-pixel refinement for previous matches; (g) UCL Gotcha based densification method for filling-in disparity gaps; (h) ASP camera triangulation and DTM creation; (i) UCL co-kriging grid-point interpolation and calculation of height uncertainties for interpolations; (j) UCL ORI co-registration/geocoding with reference to the input base map and DTM adjustment.

The CASP-GO system has recently been modified to deal with filling-in gaps in the HRSC DTM+ORI coverage of the Valles Marineris large canyon area. In a multi-stage process, which is described in detail in [3], the HRSC orbital strips are processed using CASP-GO to fill the gaps and then these are knitted into the pre-existing HRSC DTMs produced by DLR and adjusted with respect to the global MOLA mosaic reference DTM. This mosaicing process can only be applied to gap-filling and not $a b$ initio production of large-scale mosaics which require bundle block adjustment. In the process of this adjustment, errors in the HRSC orbital strip DTMs are minimised against MOLA 
removing any residual error due to spacecraft jitter. The overall processing chain of the joint 3D and image co-registration process is shown in Figure 1 and details of the final automated image-to-image co-registration and orthorectification stage in Figure 2. This has been applied to generate a 50m DTM covering the whole of Valles Marineris (see Figure 3) along with a BRDFadjusted panchromatic image mosaic at $12.5 \mathrm{~m}$ (ibid).

This new capability is planned to be incorporated from the Autumn of 2021 into the open source NASA Ames Stereo Pipeline within the joint NASA-PDART project providing the capability to the entire mapping and planetary science community to build large area datasets based upon the excellent geometrical properties of the HRSC orbital strip products aided and abetted by the MOLA gridded global reference products. This capability will also be applicable to other planetary surfaces and other non-HRSC sensors.

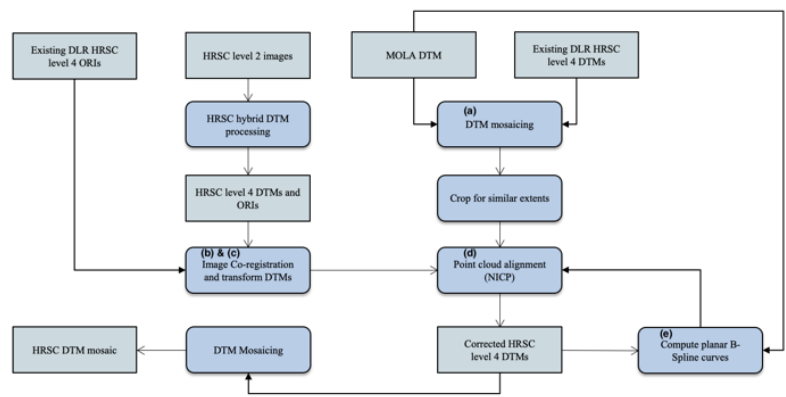

Figure 1. Flow diagram of the joint $3 D$ and image co-registration processing chain for HRSC-to-HRSC and HRSC-to-MOLA coalignment. See [3] for details of each individual component.

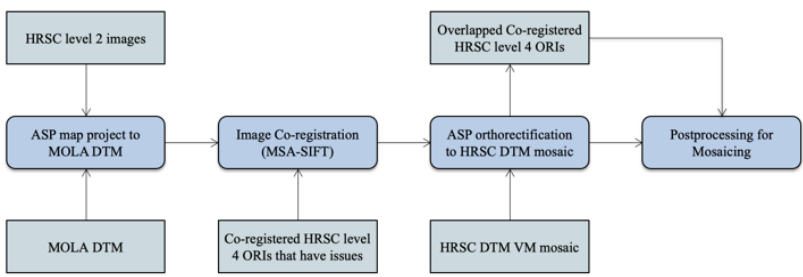

Figure 2. Flow diagram of the automated image co-registration and orthorectification processing chain. See [3] for further details.

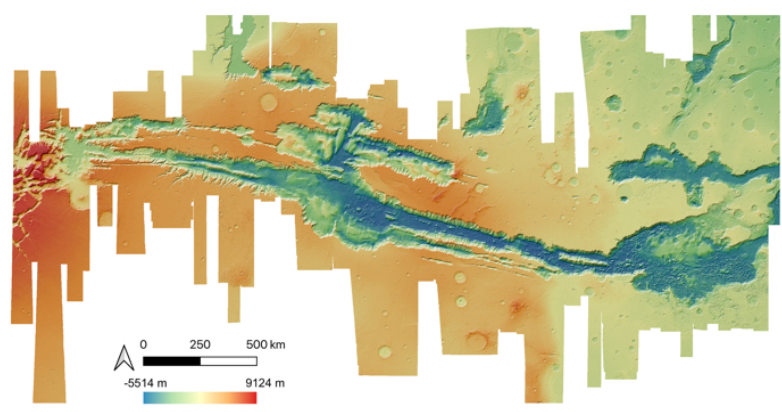

Figure 3. The final resulting HRSC DTM mosaic at 50m/pixel, covering the whole of Valles Marineris.

The CASP-GO system was optimised to be applied to stereoCTX products. In order to be able to deal with the thousands of CTX stereo-pairs and the associated Tbytes of input, intermediate and final products, the processing was run initially on a Linux server farm shown schematically in Figure 4.

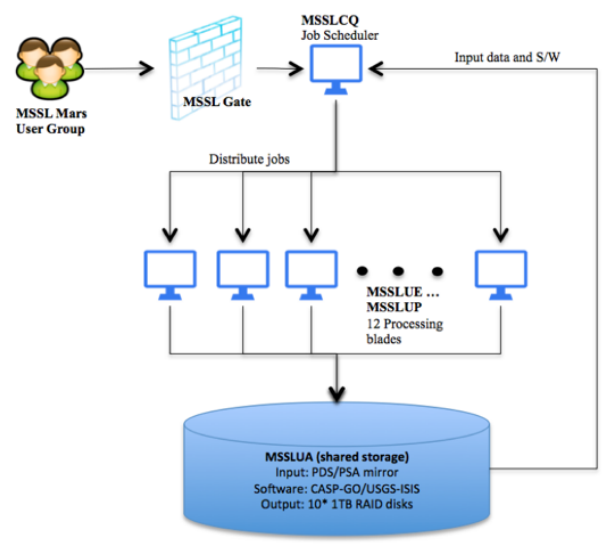

Figure 4. Schematic flowchart of the distributed linux multiblade processing system used to process the thousands of CTX stereo-pairs using CASP-GO [4]

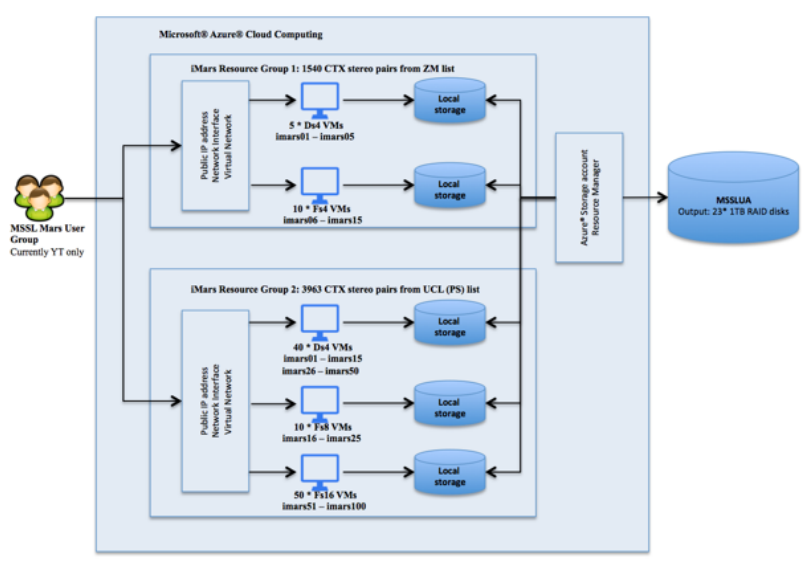

Figure 5. Microsoft ${ }^{\circledR}$ Azure ${ }^{\circledR}$ cloud computing set-up running on low specification Virtual Machines. See [4] for further details.

However, after only a few hundred scenes it became clear that much larger-scale processing was required and after successfully obtained a Microsoft $\AA$ Azure $($ cloud computing grant, the CASP-GO system was modified to process thousands of CTX stereo-pairs into DTMs and ORIs. This is shown schematically in Figure 5 for two sets of CTX stereo-pairs, firstly one set identified by members of the NASA Ames Stereo Pipeline team and then subsequently using the stereo-pairs identified by $\mathrm{P}$. Sidiropoulos (private communication, 2017).

\section{RESULTS}

After the end of the iMars project in March 2017, [4] describes how cloud computing was employed to generate some 5,300 CTX DTMs using CASP-GO. These were subsequently filtered by eliminating repeat retrievals over the same area and performing a visual analysis of the quality of DTM and ORI (OrthoRectified Image) products reduced this to 2,375 products (http://i-mars.eu/imars-products.php). All these quality controlled products are now available for direct download in geoTIFF/PVL format via the ESA Guest Storage Facility [5] landing-page at https://www.cosmos.esa.int/web/psa/UCLMSSL_iMars_CTX_v1.0 We show here some results of these products as well as more recent work on trying to generate a multi-resolution map of the vast Valles Marineris canyon system based on the HRSC basemap shown in the previous section. 


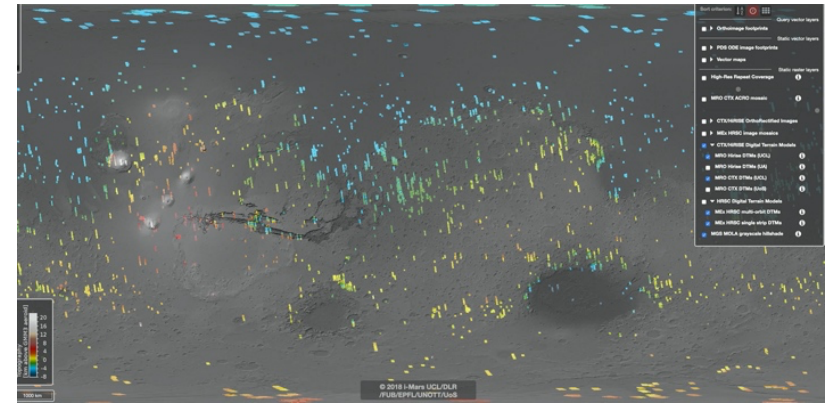

Figure 6. iMars web-GIS [6] showing distribution of 2,375 CTX $D T M+O R I$ products released through the ESA-GSF.

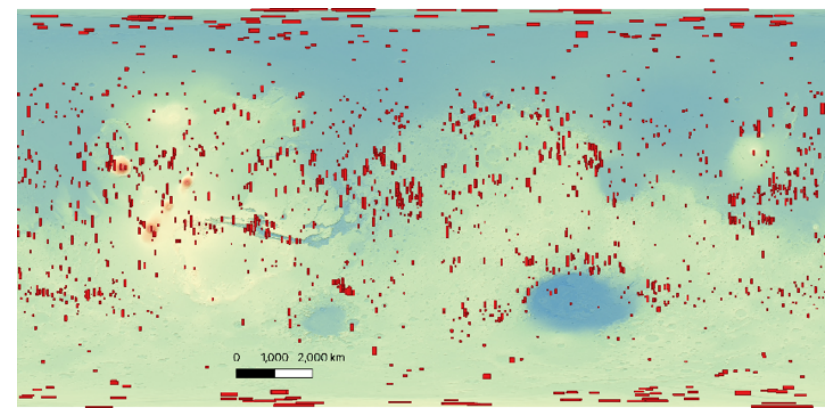

Figure 7. Distribution of 2,375 CTX 3D $18 m$ DTM and 6m ORI products covering the Martian surface.

Figure 6 and Figure 7 shows the global distribution of these $18 \mathrm{~m} /$ pixel products which are, where HRSC level-4 products exist are co-registered to the corresponding strip or mosaic HRSC DTM and ORI DLR products [6]. This figure is taken from the iMars webGIS system described in [7] and hosted at the Free University Berlin and served through the main iMars webpages at UCL-MSSL (http://www.i-mars.eu). Figure 8 and Figure 9 show two examplex of the CTX DTM products which have been hill-shaded and colourised by height intensity. They are both coregistered to HRSC ORI and co-aligned wrt their DTMs.

After this global production was completed in 2018 these 2,375 CTX 3D imaging products (doi:10.5270/esa-tk7pcfp) were released by the European Space Agency (ESA). Where possible, these CTX 3D products were co-registered to HRSC 3D products, where they had been publicly released [6]. Subsequently, very limited HiRISE DTM+ORI products [8], 35 HRSC image strips adjusted to MOLA DTM, covering the SPRC where available in the same season [9] and 82 HRSC and some 1,763 CTX stereo-pairs were processed covering the whole of the Valles Marineris area. This resulted in an 82-orbit HRSC mosaic where, in place of bundle block adjustment, a joint 3D point cloud and image co-registration using B-Spline fitting was performed to the reference MOLA DTM to eliminate both 3D shifts and high-frequency jitter effects, see previous section [3].

These DTMs and ORIs were then mosaiced (ibid) to provide a basemap for the planimetric co-registration of all CTX 3D products as well as height adjustment of all elevations to ensure plane congruence. These 524 CTX 3D products (see Figure 10) covering Valles Marineris have similar quality to what is shown in Figure 8 and Figure 9. There are still gaps remaining but these will be filled in with single-image dereived shape-from-shading results from CTX derived from the HRSC DTM using the technique described in [10].

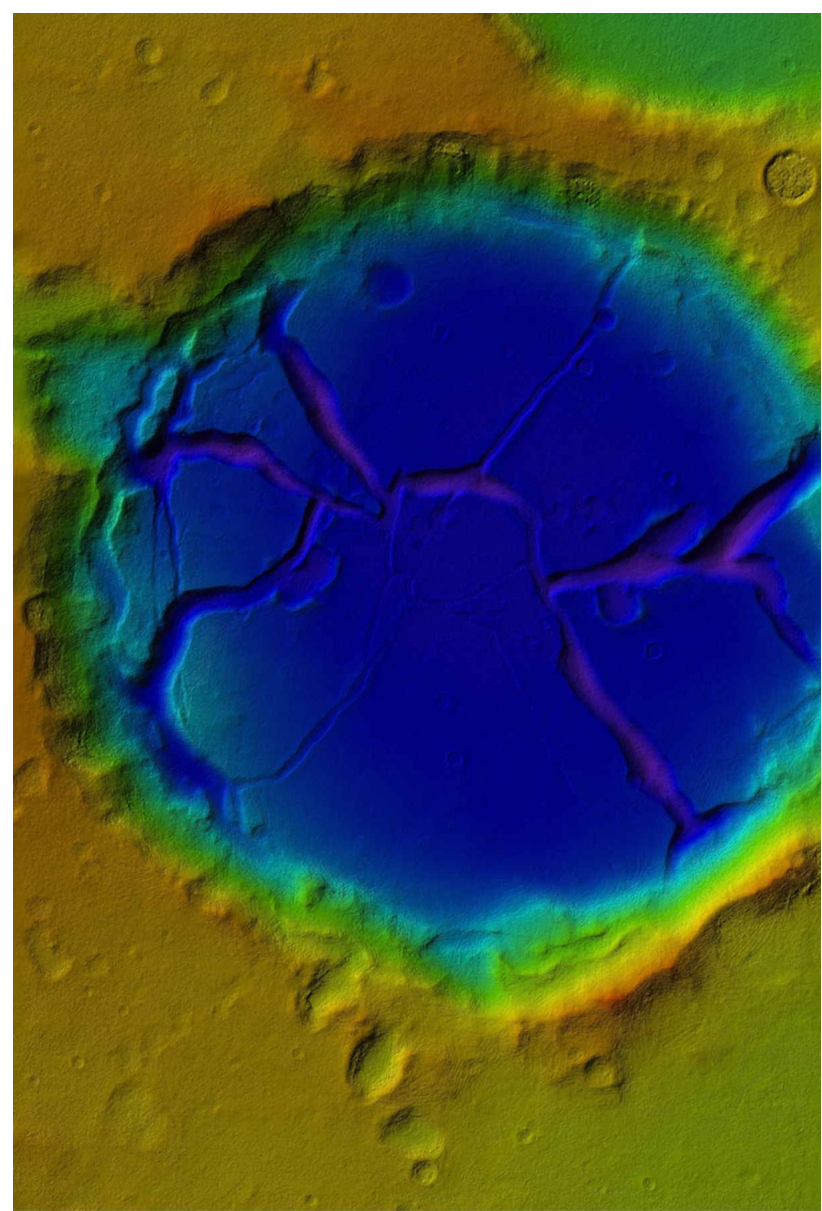

$8 \mathrm{~km}$

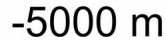

$7000 \mathrm{~m}$

Figure 8. Sample CTX DTM derived using CASP-GO created from

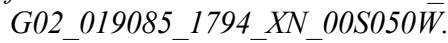
G01_018584_1794_XN_00S050W-

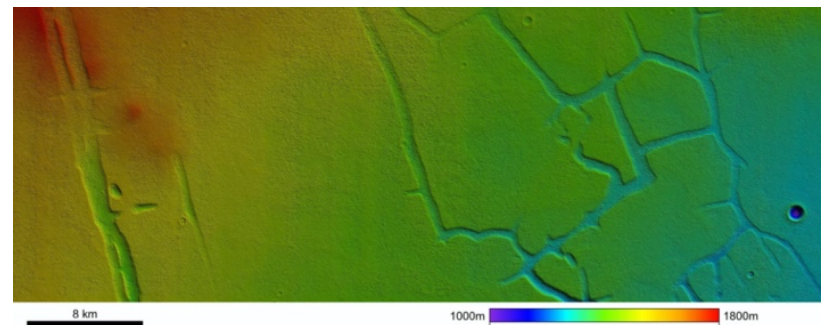

Figure 9. Example of CTX-DTM with colourisation by height and hill-shading using a standardised illumination geometry $\left(30^{\circ}\right.$ SZA and $330^{\circ}$ SAZ) generated as part of the global 2,375 set. Shown here is a DTM generated from 2 CTX level-1 images B09_013337_1847_XI_04N084W B10-013482 $1847^{-}$XI $04 N 084 \mathrm{~W}$

Subsequently, CRISM and HiRISE products are co-registered to this CTX basemap.

In Figure 10, the extent of the HRSC DTM+ORI mosaic and the co-registered CTX 3D products are shown. These products were processed using an in-house network of RHEL Linux blades with a 48-core supermicro with large RAM (384 Gb) using CASP-GO and associated ISIS functions for I/O and height adjustment. 


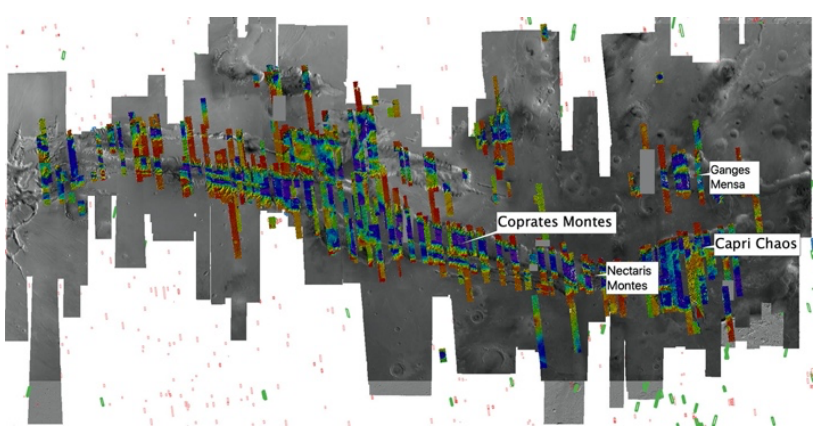

Figure 10. Map of HRSC-ORI of the panchromatic mosaic (Tao et al., in preparation) with superimposed CTX DTMs colourised by height and hill-shaded. Also shown are the footprints of HiRISE scenes (red) and CaSSIS around the perimeter (green).

All of these VM products will be publicly released via the ESAGSF. This was also performed for the iMars collection and a variety of other datasets. Browse versions will eventually be posted to the iMars webGIS [5] and linked to the original datasets. The reader is advised to consult the following URL to look at the range of Mars and lunar 3D products available (https://www.cosmos.esa.int/web/psa/ucl-mssl_meta-gsf) for download and inspection.

The afore-described multi-resolution products are being employed to provide basemap 3D imaging products for the study of Recurring Slope Lineae (RSL). As one of the key components for studying the RSL process is accurate knowledge of the slopes and aspects, we focus on multi-resolution 3D mapping of Valles Marineris, where the highest concentration of RSLs found on Mars as well as being a location where the triple point of water can be reached during the Martian summertime. For 3 selected study areas at Coprates Montes, Capri Mensa, Nectaris Montes, terrain corrected and co-registered HiRISE (at $0.25 \mathrm{~m}$ ), CRISM (at $18 \mathrm{~m}$ ) are being studied, where available.

Figure 11 shows an example, of one of the three selected study sites for RSLs in Capri Chaos, of the HRSC ORI mosaic basemap superimposed by a colourised hill-shaded $18 \mathrm{~m}$ CTX-DTM superimposed by a $0.25 \mathrm{~m}$ HiRISE ORI superimposed by 2 coregistered CRISM-FRT at $18 \mathrm{~m}$. The spatial agreement between CTX ORIs and HRSC ORI mosaic is at subpixel level of the base image $(\sim 5 \mathrm{~m})$. The vertical disagreement between CTX DTMs and HRSC/MOLA DTM mosaic was reduced from several hundreds of metres to less than $10 \mathrm{~m}$ using the proposed B-spline fitting algorithm alongside the iterative Closest Point alignment included in ASP [2]. Subsequently, we have also corrected the UoA HiRISE ORIs and DTMs using the same joint image coregistration and vertical alignment method to CTX basemap.

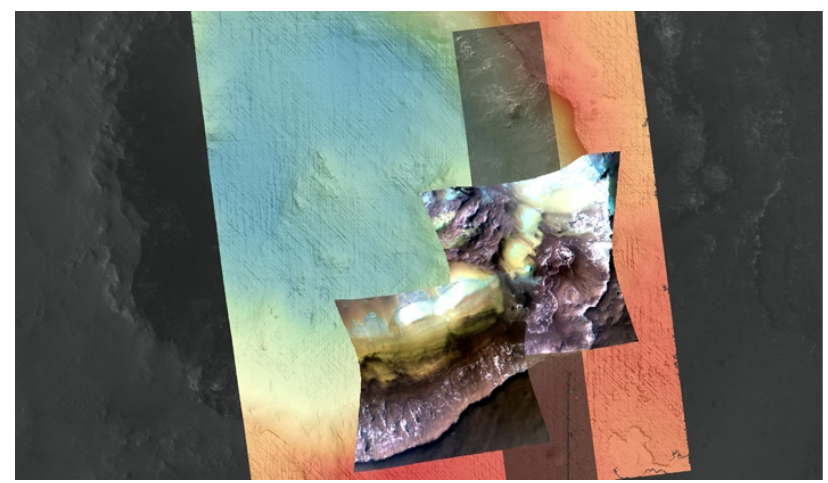

Figure 11. QGIS display of co-registered stack showing part of the HRSC ORI mosaic $12.5 \mathrm{~m}$ basemap [9] superimposed by a colourised hill-shaded $18 m$ CTX-DTM superimposed by a $0.25 \mathrm{~m}$ HiRISE ORI superimposed by 2 CRISM-FRT at $18 \mathrm{~m}$.

\section{DISCUSSION AND CONCLUSIONS}

In this work, we have outlined and demonstrated techniques and results of a 3D mosaic of the whole of the Valles Marineris area of Mars using stereo images from HRSC, as well as some examples of multi-resolution orthoimage and 3D mapping of 3 selected sites where RSLs are common, at Coprates Montes, Nectaris Montes, and Capri Chaos, using CTX, HiRISE, and CRISM. Precisely co-registered HiRISE, CRISM, CTX and HRSC data will be used as a basis for future comprehensive analyses of RSL sites using: time-series data to track RSL development, topographic analysis to constrain the slopes over which they propagate combined with information from compositional data derived from spectral and photometric data.

The CTX 3D products are all being processed into the same height reference as the HRSC basemap DTM mosaic. Shapefrom-shading techniques such as the one developed by [10] to remove artefacts and fill in gaps where no stereo retrievals were possible to achieve are being applied over a vast area. Where possible, it is planned to apply these techniques to the densification of the HRSC DTM basemap using suitable singleview CTX (ibid). This requires that the current Matlab-based technique, HDEM (ibid) can be scaled-up using GPU technology for a very large number of scenes.

\section{ACKNOWLEDGEMENTS}

The authors would like to thank the UK Space Agency for an Aurora award entitled "Understanding the role of liquids in the formation of RSLs and slope streaks within Valles Marineris using 3D super-resolution restoration" reference number ST/S001891/1. We would like to thank our colleagues on the HRSC team, especially at the Free University of Berlin and DLR for their support in the production of global HRSC orbital strips and look forward to their continuing production of global mosaics to allow the techniques shown here to be rolled out across Mars using fused HRSC-CTX-CaSSIS and HiRISE products. We would also like to thank Sebastian Walter@FUB for his unsupported maintenance of the iMars webGIS and Greg Michael@FUB for his support with the production of phaseangle corrected surface reflectance products.

\section{REFERENCES}

[1] Tao, Y., Muller, J-P., Sidiropoulos, P., Xiong, S.T., Putri, A.R.D., Walter, S.H.G., Veitch-Michaelis, J. and Yershov, V., 2018. Massive stereo-based DTM production for Mars on cloud computers. Planetary and Space Science, vol.154, pp30-58. doi: 10.1016/j.pss.2018.02.012

[2] Beyer, R., Alexandrov, O., McMichael, S. The Ames Stereo Pipeline: NASA's Opensource Software for Deriving and Processing Terrain Data. Earth Space Sci. 2018, 5, 537-548

[3] Tao, Y.; Michael, G.; Muller, J.-P.; Conway, S. J.; Putri, A. R. D. Seamless 3D Image Mapping and Mosaicing of Valles Marineris on Mars Using Orbital HRSC Stereo and Panchromatic Images. Remote Sensing 2021, 13, 1385.doi: $10.3390 /$ rs 13071385

[4] Tao, Y.; Muller, J.-P., 2018. Performance of global 3D model retrievals of the Martian surface using the UCL CASP-GO system on CTX stereo images on linux clusters and Microsoft 
Azure ${ }^{\circledR}$ cloud computing platforms. In; Huang, B.; López, S.; Wu, Z., Eds.; SPIE, 2018; v.10792, p.7.doi: 10.1117/12.2500195

[5] Muller, J.-P., Tao, Y., A.R.D. Putri1, G. Watson, R. Beyer, O. Alexandrov, S. McMichael, S. Besse, E. Grotheer. 3D imaging tools and geospatial services from joint European-USA collaborations. EPSC-DPS conference, 2020, vol. 13, EPSCDPS2019-1355-1.doi: 10.5270/esa-0j79yk8

[6] Gwinner, K.; Jaumann, R.; Hauber, E.; HOFFMANN, H.; Heipke, C.; Oberst, J.; Neukum, G.; Ansan, V.; Bostelmann, J.; Dumke, A.; Elgner, S.; Erkeling, G.; Fueten, F.; Hiesinger, H.; Hoekzema, N. M.; Kersten, E.; Loizeau, D.; Matz, K.-D.; McGuire, P. C.; Mertens, V.; Michael, G.; Pasewaldt, A.; Pinet, P.; Preusker, F.; Reiss, D.; Roatsch, T.; Schmidt, R.; Scholten, F.; Spiegel, M.; Stesky, R.; Tirsch, D.; van Gasselt, S.; Walter, S.; Wahlisch, M.; Willner, K. The High Resolution Stereo Camera (HRSC) of Mars Express and its approach to science analysis and mapping for Mars and its satellites. Planetary and Space Science 2016, 126, 93-138.

[7] Walter, S.H.G., Muller, J-P., Sidiropoulos, P., Tao, Y., Gwinner, K., Putri, A.R.D., Kim, J.R., Steikert, R., Gasselt, S.V., Michael, G.G. and Watson, G., 2018. The iMars web GIS-an interactive online mapping tool for the spatio-temporal visualization of topography data and dynamic time-series of single image layers. JGR/ESS special issue: Planetary Mapping: Methods, Tools for Scientific Analysis and Exploration, vol. 5, pp 308-323. doi: 10.1029/2018EA000389

[8] Xiong, S.; Tao, Y.; Persaud, D. M.; Campbell, J. D.; Diana Putri, A. R.; Muller, J.-P. Subsurface Reflectors Detected by SHARAD Reveal Stratigraphy and Buried Channels over Central Elysium Planitia, Mars. Earth and Space Science 2020, 7, e2019EA000968.doi: 10.1029/2019EA000968

[9] Putri, A. R. D.; Sidiropoulos, P.; Muller, J.-P.; Walter, S. H. G.; Michael, G. G. A New South Polar Digital Terrain Model of Mars from the High Resolution Stereo Camera (HRSC) onboard the ESA Mars Express. Planetary and Space Science 2019, 174, 43-55.doi: 10.1016/j.pss.2019.02.010

[10] Douté, S.; Jiang, C. Small-Scale Topographical Characterization of the Martian Surface With In-Orbit Imagery. IEEE Trans. Geosci. Rem. Sens. 2020, 58, 447-460.doi: 10.1109/TGRS.2019.2937172 